Homology, Homotopy and Applications, vol.21(2), 2019, pp.199-212

\title{
A MOMENT-ANGLE MANIFOLD WHOSE COHOMOLOGY HAS TORSION
}

\author{
XIAOMENG LI AND GEFEI WANG
}

(communicated by Nicholas J. Kuhn)

\begin{abstract}
In this paper we give a method to construct moment-angle manifolds whose cohomology has torsion. We also give method to describe the corresponding simplicial sphere by its non-faces.
\end{abstract}

\section{Introduction}

Corresponding to every abstract simplicial complex $L$ on the vertex set $[m]=$ $\{1,2, \ldots, m\}$, there are the real and complex moment-angle complexes $\mathbb{R} \mathcal{Z}_{L}$ and $\mathcal{Z}_{L}$ $(c f .[3,4])$. They are defined as

$$
\begin{aligned}
\mathbb{R} \mathcal{Z}_{L} & =\bigcup_{\sigma \in L} \prod_{i \in \sigma} D_{i}^{1} \times \prod_{i \notin \sigma} S_{i}^{0} \subseteq D_{1}^{1} \times D_{2}^{1} \times \cdots \times D_{m}^{1}, \\
\mathcal{Z}_{L} & =\bigcup_{\sigma \in L} \prod_{i \in \sigma} D_{i}^{2} \times \prod_{i \notin \sigma} S_{i}^{1} \subseteq D_{1}^{2} \times D_{2}^{2} \times \cdots \times D_{m}^{2} .
\end{aligned}
$$

The cohomology groups of $\mathbb{R} \mathcal{Z}_{L}$ and $\mathcal{Z}_{L}$ are given by Hochster's theorem: Corresponding to every abstract simplicial complex $L$ on the vertex set $[m]=\{1,2, \ldots, m\}$, there are the real and complex moment-angle complexes $\mathbb{R} \mathcal{Z}_{L}$ and $\mathcal{Z}_{L}(c f .[\mathbf{3}, \mathbf{4}])$. They are defined as

$$
\begin{aligned}
\mathbb{R} \mathcal{Z}_{L} & =\bigcup_{\sigma \in L} \prod_{i \in \sigma} D_{i}^{1} \times \prod_{i \notin \sigma} S_{i}^{0} \subseteq D_{1}^{1} \times D_{2}^{1} \times \cdots \times D_{m}^{1}, \\
\mathcal{Z}_{L} & =\bigcup_{\sigma \in L} \prod_{i \in \sigma} D_{i}^{2} \times \prod_{i \notin \sigma} S_{i}^{1} \subseteq D_{1}^{2} \times D_{2}^{2} \times \cdots \times D_{m}^{2} .
\end{aligned}
$$

The cohomology groups of $\mathbb{R} \mathcal{Z}_{L}$ and $\mathcal{Z}_{L}$ are given by Hochster's theorem:

Theorem $1.1([\mathbf{1}, \mathbf{3}, \mathbf{4}])$. Let $L$ be a simplicial complex on the vertex set $[m]$, then

$$
\begin{aligned}
H^{*}\left(\mathbb{R} \mathcal{Z}_{L}\right) & \cong \bigoplus_{I \subset[m]} \widetilde{H}^{*-1}\left(\left.L\right|_{I}\right), \\
H^{*}\left(\mathcal{Z}_{L}\right) & \cong \bigoplus_{I \subset[m]} \widetilde{H}^{*-|I|-1}\left(\left.L\right|_{I}\right),
\end{aligned}
$$

\footnotetext{
This project is supported by NSFC No. 11471167, No. 11871284 and No. 11761072.

Received July 3, 2018, revised September 26, 2018; published on February 27, 2019.

2010 Mathematics Subject Classification: 13F55, 05A19, 05E40, 52B05, 52B10.

Key words and phrases: moment-angle manifold, stellar subdivision, full subcomplex, missing face and simplicial complement.

Article available at http://dx.doi.org/10.4310/HHA.2019.v21.n2.a11

Copyright (C) 2019, International Press. Permission to copy for private use granted.
} 
where $\left.L\right|_{I}$ is the full subcomplex of $L$ on subset $I$ and $I$ runs over all the subsets of $[m]$.

From $[\mathbf{5}, \mathbf{6}, \mathbf{7}]$ we know that both $\mathbb{R} \mathcal{Z}_{L}$ and $\mathcal{Z}_{L}$ are topological manifolds if $L$ is a simplicial sphere, referred to as moment-angle manifolds. Furthermore if $L$ is a polytopal sphere (the boundary complex of a simplicial polytope), then $\mathcal{Z}_{L}$ is a transverse intersection of real quadratic hypersurfaces ( $c f .[\mathbf{2}])$, while both $\mathbb{R} \mathcal{Z}_{L}$ and $\mathcal{Z}_{L}$ are framed differentiable manifolds.

Bosio and Meersseman in [2] announced that the cohomology groups of differentiable complex moment-angle manifolds may have any torsion $\mathbb{Z} / m$. Furthermore if $L$ is $\mathbb{Z} / 2$ colourable, Cai, Choi and Park in $[\mathbf{8}, \mathbf{9}]$ proved that the small cover under $\mathbb{R} \mathcal{Z}_{L}$ may have any torsion $\mathbb{Z} / m$.

From Hochster's theorem, it is easy to construct a moment-angle complex whose cohomology has torsion. But it is harder to construct such moment-angle manifolds, at least, the cohomology of all the moment-angle manifolds corresponding to dimensional 1, 2 and 3 simplicial spheres are torsion free ( $c f$. [2, Corollary 11.1]).

Based on Hochster's theorem, our goal is to find a simplicial complex $K$ whose cohomology has torsion and $K$ is embedded in a polytopal sphere $L$ as a full subcomplex. Then both the real and complex moment-angle complexes corresponding to $L$ are differentiable manifolds and the cohomology of $\mathbb{R} \mathcal{Z}_{L}$ and $\mathcal{Z}_{L}$ have $\tilde{H}^{*}(K)$ as a summand and then have torsion.

Theorem 3.2 (Construction). Let $K$ be a subcomplex (not a full subcomplex) of a simplicial sphere $L_{0}$ on the vertex set $[m], \mathbb{M}=\left\{\sigma_{1}, \sigma_{2}, \ldots, \sigma_{s}\right\}$ be the set of missing faces of $K$, which are also simplices of $L_{0}$. On $L_{0}$, make stellar subdivisions at $\sigma_{1}, \sigma_{2}, \ldots, \sigma_{s}$ one by one as follows

$$
L_{1}=s s_{\sigma_{1}} L_{0}, \quad L_{2}=s s_{\sigma_{2}} L_{1}, \quad \ldots, \quad L_{s}=s s_{\sigma_{s}} L_{s-1} .
$$

Then $K$ becomes a full subcomplex of $L_{s}, K=\left.L_{s}\right|_{[m]}$.

In fact, after making stellar subdivision on a polytopal (simplicial) sphere, it is still polytopal (simplicial) (see [10]). If $L_{0}$ is also a polytopal sphere, we thus obtain a polytopal sphere $L_{s}$ by Theorem 3.2 such that $K$ is a full subcomplex of $L_{s}$. The real and complex moment-angle complexes corresponding to $L_{s}$ are differentiable manifolds. By Hochster's theorem both $H^{*}\left(\mathbb{R}_{L_{s}}\right)$ and $H^{*}\left(\mathcal{Z}_{L_{s}}\right)$ have torsion if $\widetilde{H}^{*}(K)$ has torsion.

At last in Section 4, we give a differentiable moment-angle manifold whose cohomology has $\mathbb{Z} / 3$ as a summand. This is done as follows:

Triangulate the mod 3 Moore space $K$ which has 8 vertices, 172 -dimensional facets and 22 missing faces (see Figure 3). It can be embedded in $\partial \Delta^{7}=L_{0}$. After making 22 stellar subdivisions on it, $K$ becomes a full subcomplex of the polytopal sphere $L_{22}$. Then $L_{22}$ is a 6 -dimensional polytopal sphere with 30 vertices. $\mathcal{Z}_{L_{22}}$ is a 37 dimensional differentiable manifold and $H^{11}\left(\mathcal{Z}_{L_{22}}\right)$ has $\widetilde{H}^{2}(K)=\mathbb{Z} / 3$ as a summand.

It is notable that Bosio and Meersseman's construction in [2, Theorem 11.12] applied to the same example does not give a moment-angle manifold whose cohomology has $\mathbb{Z} / 3$ torsion. 


\section{Acknowledgments}

The authors are grateful to Professor Zhi Lü for his helpful suggestion during this research. This work was done under the supervision of Professor Xiangjun Wang.

\section{Simplicial complement}

An abstract simplicial complex $K$ on the vertex set $I$ is a collection of simplices that satisfies: for any simplex (face) $\sigma \in K$, all of its proper subsets (proper faces) are simplices of $K$.

An abstract simplicial complex $K$ could also be given by all of its non-faces

$$
\mathbb{A}=2^{I} \backslash K
$$

and

$$
K=2^{I} \backslash \mathbb{A}
$$

that satisfies: if $\sigma \in \mathbb{A}$ is not a simplex of $K$ and $\sigma^{\prime} \supset \sigma$ then $\sigma^{\prime} \in \mathbb{A}$ is not a simplex of $K$.

A simplex $\sigma=\left(i_{1}, i_{2}, \ldots, i_{k}\right) \in 2^{I}$ is called a missing face (or minimal non-face) of $K$ if it is not a face of $K$, but all of its proper subsets are faces of $K$, i.e. $\sigma \notin K$ but every $\sigma_{j}=\left(i_{1}, \ldots, \widehat{i_{j}}, \ldots, i_{k}\right) \in K, j=1,2, \ldots, k$. An abstract simplicial complex could also be given by its set of missing faces

$$
\mathbb{M}=\left\{\sigma \in 2^{I} \mid \sigma \text { is a missing face of } K\right\}
$$

and

$$
K=\left\{\tau \in 2^{I} \mid \tau \text { does not contain any } \sigma \in \mathbb{M}\right\} .
$$

A subset $\sigma^{\prime}$ of $I$ is not a simplex of $K$ if and only if it contains a missing face $\sigma \in \mathbb{M}$ as a subset.

Definition 2.1. Let $K$ be a simplicial complex on the vertex set $I$ and $\mathbb{M}, \mathbb{A}$ be the sets of missing faces and non-faces of $K$ respectively. We define a simplicial complement of $K$, denoted by

$$
\mathbb{P}=\left\{\sigma_{1}, \sigma_{2}, \ldots, \sigma_{s}\right\},
$$

to be a collection of non-faces that includes all the missing faces $\mathbb{M}$ i.e.

$$
\mathbb{M} \subseteq \mathbb{P} \subseteq \mathbb{A} \text {. }
$$

Similar to the set of missing faces $\mathbb{M}$, given a simplicial complement $\mathbb{P}$ (collection of non-faces) on the vertex set $I$, one can obtain a simplicial complex $K_{\mathbb{P}}$ on $I$ by:

$$
K_{\mathbb{P}}(I)=\left\{\tau \subset I \mid \tau \text { does not contain any } \sigma_{i} \in \mathbb{P}\right\}
$$

or by all of its non-faces

$$
2^{I} \backslash K_{\mathbb{P}}(I)=\left\{\tau \subset I \mid \tau \text { contains a } \sigma_{i} \in \mathbb{P}\right\} .
$$

A subset $\sigma$ of $I$ is not a simplex of $K_{\mathbb{P}}(I)$ if and only if it contains a non-face $\sigma_{i}$ in the simplicial complement $\mathbb{P}$. 
Definition 2.2. Let $\mathbb{P}, \mathbb{P}^{\prime}$ be two simplicial complements on the vertex set $I$, if they can obtain the same simplicial complex i.e. $K_{\mathbb{P}}(I)=K_{\mathbb{P}^{\prime}}(I)$, we say that $\mathbb{P}$ and $\mathbb{P}^{\prime}$ are equivalent, denoted by $\mathbb{P} \simeq \mathbb{P}^{\prime}$.

It is easy to see that: Two simplicial complements $\mathbb{P}, \mathbb{P}^{\prime}$ on $I$ are equivalent if and only if for every non-face $\sigma \in \mathbb{P}$ there exists a $\sigma^{\prime} \in \mathbb{P}^{\prime}$ such that $\sigma^{\prime} \subseteq \sigma$ and for every non-face $\sigma^{\prime} \in \mathbb{P}^{\prime}$ there exists a $\sigma \in \mathbb{P}$ such that $\sigma \subseteq \sigma^{\prime}$.

Proposition 2.3. Let $\mathbb{P}=\left\{\sigma_{1}, \sigma_{2}, \ldots, \sigma_{s}\right\}$ be a simplicial complement of $K$ on $I$. For a non-face $\sigma_{j} \in \mathbb{P}$ if there exists a $\sigma_{i} \in \mathbb{P}, i \neq j$ such that $\sigma_{i} \subseteq \sigma_{j}$, then we can remove $\sigma_{j}$ from $\mathbb{P}$ and the resulting simplicial complement

$$
\mathbb{P}^{\prime}=\left\{\sigma_{1}, \sigma_{2}, \ldots, \widehat{\sigma}_{j}, \ldots, \sigma_{s}\right\}
$$

is equivalent to $\mathbb{P}$. In this case we call that $\mathbb{P}$ is reduced to $\mathbb{P}^{\prime}$.

Every simplicial complement of $K$ could be reduced to the set of missing faces by removing all the larger non-faces.

Example 2.4. The simplicial complex $K$ is determined by the maximal simplices $(1,3),(2,3),(1,2,4),(1,2,5),(1,4,5),(2,4,5)$ and their proper subsets on the vertex set $[5]=\{1,2,3,4,5\}$ (see Figure 1)

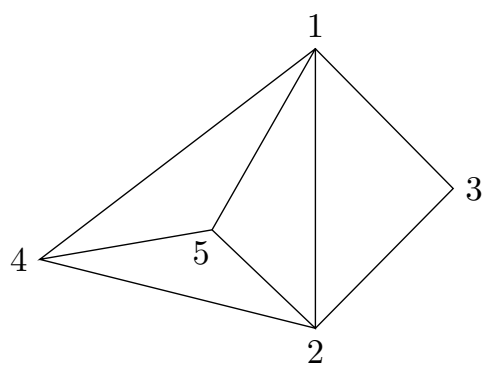

Figure 1: The simplicial complex $K$

$$
\mathbb{P}=\left\{\begin{array}{l}
\sigma_{1}=(1,2,4,5), \sigma_{2}=(1,2,3), \sigma_{3}=(3,4), \\
\sigma_{4}=(3,5), \sigma_{5}=(1,3,4), \sigma_{6}=(3,4)
\end{array}\right\}
$$

is a simplicial complement of $K$ on the vertex set [5] where $\sigma_{3}=(3,4)=\sigma_{6}$ appeared twice and $\sigma_{3}=(3,4) \subset \sigma_{5}=(1,3,4)$. So $\sigma_{5}=(1,3,4)$ and $\sigma_{6}=(3,4)$ could be removed from $\mathbb{P}$ to reduce to the set of missing faces $\mathbb{M}=\{(1,2,4,5),(1,2,3),(3,4)$, $(3,5)\}$.

The readers should be aware that the empty simplex $\{\emptyset\}$ (only the empty set is a simplex) is different from the empty complex $\emptyset$ (the empty set is not a simplex of $\emptyset$ ). $\mathbb{M}=\{(1),(2), \ldots,(m)\}$ is the set of missing faces of the empty simplex $\{\emptyset\}$ while $\mathbb{M}_{1}=\{\emptyset\}$ is the set of missing faces of the empty complex $\emptyset$. 
Let $K$ be a simplicial complex on the vertex set $I$ and $\sigma$ be a simplex of $K$. The link and star of $\sigma$ are defined to be the simplicial complexes

$$
\operatorname{link}_{K} \sigma=\{\tau \in K \mid \sigma \cup \tau \in K, \sigma \cap \tau=\emptyset\}, \quad \operatorname{star}_{K} \sigma=\{\tau \in K \mid \sigma \cup \tau \in K\} .
$$

The interior (open) star is defined to be the set of simplices (do not contain their proper faces)

$$
\text { Intstar }_{K} \sigma=\{\tau \in K \mid \sigma \subset \tau\}
$$

and the boundary of star is the simplicial complex (cf. [12, Lemma 62.6])

$$
\partial \operatorname{star}_{K} \sigma=\operatorname{star}_{K} \sigma \backslash \text { Intstar }_{K} \sigma=\{\tau \in K \mid \sigma \cup \tau \in K, \sigma \not \subset \tau\} .
$$

Let $K_{1}$ and $K_{2}$ be two simplicial complexes on the vertex set $I$ and $J$, where $I \cap J=\emptyset$. The join of $K_{1}$ and $K_{2}$ is defined to be the simplicial complex on the vertex set $I \cup J$

$$
K_{1} * K_{2}=\left\{\sigma \cup \tau \in 2^{I \cup J} \mid \sigma \in K_{1}, \tau \in K_{2}\right\} .
$$

Let $\mathbb{P}=\left\{\sigma_{1}, \sigma_{2}, \ldots, \sigma_{s}\right\}$ be a simplicial complement of $K$ on the vertex set $I$ and $\sigma \in K$ be a simplex. We define

$$
\mathbb{P}-\sigma=\left\{\sigma_{1} \backslash \sigma, \sigma_{2} \backslash \sigma, \ldots, \sigma_{s} \backslash \sigma\right\}
$$

which is a sequence of subsets on $I \backslash \sigma$.

Lemma 2.5. Let $\mathbb{P}=\left\{\sigma_{1}, \sigma_{2}, \ldots, \sigma_{s}\right\}$ be a simplicial complement of $K$ on the vertex set I. Then

1. $\mathbb{P}-\sigma=\left\{\sigma_{1} \backslash \sigma, \sigma_{2} \backslash \sigma, \ldots, \sigma_{s} \backslash \sigma\right\}$ is a simplicial complement of link $k_{K} \sigma$ on the vertex set $I \backslash \sigma$, i.e. by (1)

$\operatorname{link}_{K} \sigma=K_{\mathbb{P}-\sigma}(I \backslash \sigma)=\left\{\tau \subset(I \backslash \sigma) \mid \sigma\right.$ does not contain any $\left.\sigma_{i} \backslash \sigma \in \mathbb{P}-\sigma\right\}$.

2. If we consider $\mathbb{P}-\sigma$ as a sequence of non-faces on the vertex set $I$, then it is a simplicial complement of $\operatorname{star}_{K} \sigma$ on I, i.e. by (1)

$$
\text { star }_{K} \sigma=K_{\mathbb{P}-\sigma}(I)=\left\{\tau \subset I \mid \sigma \text { does not contain any } \sigma_{i} \backslash \sigma \in \mathbb{P}-\sigma\right\} .
$$

Proof. We prove this lemma by showing that they have the same non-faces

$$
2^{I \backslash \sigma} \backslash \operatorname{link}_{K} \sigma=2^{I \backslash \sigma} \backslash K_{\mathbb{P}-\sigma}(I \backslash \sigma)=\left\{\tau \subset(I \backslash \sigma) \mid \tau \text { contains a } \sigma_{i} \backslash \sigma \in \mathbb{P}-\sigma\right\}
$$

and

$$
2^{I} \backslash \operatorname{star}_{K} \sigma=2^{I} \backslash K_{\mathbb{P}-\sigma}(I)=\left\{\tau \subset I \mid \tau \text { contains a } \sigma_{i} \backslash \sigma \in \mathbb{P}-\sigma\right\} .
$$

1. From its definition, we know that a simplex $\tau$ on the vertex set $I \backslash \sigma$ is not a simplex of $\operatorname{link}_{K} \sigma$ if and only if $\sigma \cup \tau$ is not a simplex of $K$. In other words, there exists a $\sigma_{i} \in \mathbb{P}$ such that $\sigma_{i} \subseteq \tau \cup \sigma$. This is equivalent to say that $\sigma_{i} \backslash \sigma \subseteq$ $\tau \cup \sigma \backslash \sigma=\tau$, every non-face $\tau \in 2^{I \backslash \sigma} \backslash \operatorname{link}_{K} \sigma$ is a non-face of $K_{\mathbb{P}-\sigma}(I \backslash \sigma)$, i.e. $\tau \in 2^{I \backslash \sigma} \backslash K_{\mathbb{P}-\sigma}(I \backslash \sigma)$, so

$$
2^{I \backslash \sigma} \backslash \operatorname{link}_{K} \sigma \subseteq 2^{I \backslash \sigma} \backslash K_{\mathbb{P}-\sigma}(I \backslash \sigma) .
$$

2. If a simplex $\tau$ on the vertex set $I \backslash \sigma$ contains a $\sigma_{i} \backslash \sigma$, then $\tau \cup \sigma \supseteq\left(\sigma_{i} \backslash \sigma\right) \cup$ $\sigma \supseteq \sigma_{i}$, so such $\tau$ is not a simplex of $\operatorname{link}_{K} \sigma$. This is equivalent to say that every 
non-face $\tau \in 2^{I \backslash \sigma} \backslash K_{\mathbb{P}-\sigma}(I \backslash \sigma)$ is a non-face of $\operatorname{link}_{K} \sigma$, i.e. $\tau \in 2^{I \backslash \sigma} \backslash \operatorname{lin} k_{K} \sigma$, so

$$
2^{I \backslash \sigma} \backslash K_{\mathbb{P}-\sigma}(I \backslash \sigma) \subseteq 2^{I \backslash \sigma} \backslash \operatorname{link}_{K} \sigma .
$$

Thus $\mathbb{P}-\sigma=\left\{\sigma_{1} \backslash \sigma, \sigma_{2} \backslash \sigma, \ldots, \sigma_{s} \backslash \sigma\right\}$ is a simplicial complement of $\operatorname{link}_{K} \sigma$ on the vertex set $I \backslash \sigma$.

Similarly, if we consider $\mathbb{P}-\sigma$ as a simplicial complement on the vertex set $I$, then

$$
2^{I} \backslash \operatorname{star}_{K} \sigma=2^{I} \backslash K_{\mathbb{P}-\sigma}(I)=\left\{\tau \subset I \mid \tau \text { contains a } \sigma_{i} \backslash \sigma \in \mathbb{P}-\sigma\right\} .
$$

The lemma follows.

Example 2.6. In Example 2.4, the link of the simplex $(1,2)$ is the pair of vertices $\operatorname{link}_{K}(1,2)=\{(4),(5)\}$ and $\operatorname{star}_{K}(1,2)$ is composed of two 2-simplices $(1,2,4)$, $(1,2,5)$ and its proper subsets.

$$
\begin{aligned}
\mathbb{M}-(1,2) & =\{(1,2,4,5) \backslash(1,2), \quad(1,2,3) \backslash(1,2), \quad(3,4) \backslash(1,2), \quad(3,5) \backslash(2)\} \\
& =\{(4,5),(3),(3,4),(3,5)\} \\
& \simeq\{(4,5),(3)\}
\end{aligned}
$$

is a simplicial complement of $\operatorname{link}_{K}(1,2)$ on the vertex set $\{3,4,5\}$. Consider $\mathbb{M}-\sigma$ as a sequence of non-faces on the vertex set $[5]=\{1,2,3,4,5\}$, it becomes the simplicial complement of $\operatorname{star}_{K}(1,2)$.

Let $\mathbb{P}_{1}=\left\{\sigma_{1}, \sigma_{2}, \ldots, \sigma_{s}\right\}$ and $\mathbb{P}_{2}=\left\{\tau_{1}, \tau_{2}, \ldots, \tau_{t}\right\}$ be the simplicial complements of $K_{1}$ and $K_{2}$ on the vertex set $I$. We define their join $\mathbb{P}_{1} * \mathbb{P}_{2}$ to be

$$
\mathbb{P}_{1} * \mathbb{P}_{2}=\left\{\sigma_{i} \cup \tau_{j} \mid \sigma_{i} \in \mathbb{P}_{1}, \tau_{j} \in \mathbb{P}_{2}\right\},
$$

which is a sequence of subsets on $I$.

Lemma 2.7. Let $K_{1}$ and $K_{2}$ be two simplicial complexes on the vertex set $I, \mathbb{P}_{1}=$ $\left\{\sigma_{1}, \sigma_{2}, \ldots, \sigma_{s}\right\}$ and $\mathbb{P}_{2}=\left\{\tau_{1}, \tau_{2}, \ldots, \tau_{t}\right\}$ be simplicial complements of $K_{1}$ and $K_{2}$ respectively. Then $\mathbb{P}_{1} * \mathbb{P}_{2}=\left\{\sigma_{i} \cup \tau_{j} \mid \sigma_{i} \in \mathbb{P}_{1}, \tau_{j} \in \mathbb{P}_{2}\right\}$ is a simplicial complement of $K_{1} \cup K_{2}$ on the vertex set $I$,

$$
K_{1} \cup K_{2}=K_{\mathbb{P}_{1} * \mathbb{P}_{2}}(I)=\left\{\tau \subset I \mid \tau \text { does not contain any } \sigma_{i} \cup \tau_{j} \in \mathbb{P}_{1} * \mathbb{P}_{2}\right\} .
$$

Proof. We prove this lemma in the same way as the proof of Lemma 2.5.

1. It is easy to see that a simplex $\tau$ on the vertex set $I$ is not a simplex of $K_{1} \cup K_{2}$ if and only if it is not a simplex of either $K_{1}$ or $K_{2}$. This implies that there exists a $\sigma_{i} \in \mathbb{P}_{1}$ such that $\sigma_{i} \subseteq \tau$ and also exists a $\tau_{j} \in \mathbb{P}_{2}$ such that $\tau_{j} \subseteq \tau$. This is equivalent to say that $\sigma_{i} \cup \tau_{j} \subseteq \tau$, every non-face $\tau$ of $K_{1} \cup K_{2}$ contains a $\sigma_{i} \cup \tau_{j} \in \mathbb{P}_{1} * \mathbb{P}_{2}$, so

$$
2^{I} \backslash K_{1} \cup K_{2} \subseteq 2^{I} \backslash K_{\mathbb{P}_{1} * \mathbb{P}_{2}}(I) .
$$

2. If a simplex $\tau$ on $I$ contains a non-face $\sigma_{i} \cup \tau_{j} \in \mathbb{P}_{1} * \mathbb{P}_{2}$, then $\sigma_{i} \subseteq \tau$ and $\tau_{j} \subseteq \tau$. This $\tau$ is neither a simplex of $K_{1}$ nor a simplex of $K_{2}$, so

$$
2^{I} \backslash K_{\mathbb{P}_{1} * \mathbb{P}_{2}}(I) \subseteq 2^{I} \backslash K_{1} \cup K_{2} .
$$

The lemma follows. 
Corollary 2.8. If the simplicial complement $\mathbb{P}$ is equivalent to $\mathbb{P}^{\prime}$, then for any simplex $\sigma$ and simplicial complement $\mathbb{P}_{2}$

$$
\mathbb{P}-\sigma \simeq \mathbb{P}^{\prime}-\sigma, \quad \mathbb{P} * \mathbb{P}_{2} \simeq \mathbb{P}^{\prime} * \mathbb{P}_{2} .
$$

Let $\sigma$ be a simplex of a simplicial complex $K$ on $[m]$. The stellar subdivision at $\sigma$ on $K$ is defined to be the union of the simplicial complexes $K \backslash \operatorname{Intstar}_{K} \sigma$ and the cone conedstar ${ }_{K} \sigma$ along their boundary $\partial s t a r_{K} \sigma$, denoted by

$$
s s_{\sigma} K=\left(K \backslash \text { Intstar }_{K} \sigma\right) \cup\left(\text { conedstar }_{K} \sigma\right),
$$

where

$$
K \backslash \operatorname{Intstar}_{K} \sigma=\{\tau \in K \mid \sigma \not \subset \tau\}
$$

and

$$
\operatorname{cone} \operatorname{star}_{K} \sigma=(m+1) * \partial \operatorname{star}_{K} \sigma .
$$

After stellar subdivision, one more vertex is added which is the vertex of the cone (cf. $[\mathbf{2}])$.

In [4, Definition 2.7.1], the stellar subdivision is defined to be

$$
s s_{\sigma} K=\left(K \backslash \operatorname{star}_{K} \sigma\right) \cup\left(\text { conedstar }_{K} \sigma\right),
$$

where $K \backslash \operatorname{star}_{K} \sigma$ is not a simplicial complex. Note that

$$
K \backslash \operatorname{star}_{K} \sigma=\left(K \backslash \text { Intstar }_{K} \sigma\right) \backslash \partial \operatorname{star}_{K} \sigma
$$

and

$$
\left(K \backslash \operatorname{Intstar}_{K} \sigma\right) \cap\left(\text { conedstar }_{K} \sigma\right)=\partial \operatorname{star}_{K} \sigma,
$$

so our definition coincides with that in [4].

Theorem 2.9. Let $\mathbb{P}=\left\{\sigma_{1}, \sigma_{2}, \ldots, \sigma_{s}\right\}$ be a simplicial complement of $K$. Then $\{\mathbb{P}, \sigma,(\mathbb{P}-\sigma) *(m+1)\}$ is a simplicial complement of $s s_{\sigma} K$ on the vertex set $[m+1]$, where

$$
(\mathbb{P}-\sigma) *(m+1)=\left\{\left(\sigma_{1} \backslash \sigma, m+1\right),\left(\sigma_{2} \backslash \sigma, m+1\right), \ldots,\left(\sigma_{s} \backslash \sigma, m+1\right)\right\} .
$$

Proof. First we prove that $\{\sigma, \mathbb{P}-\sigma\}$ is a simplicial complement of conedstar ${ }_{K} \sigma$ on the vertex set $[m+1]=\{1,2, \ldots, m, m+1\}$.

From Lemma 2.5 we know that $\mathbb{P}-\sigma$ is a simplicial complement of $\operatorname{star}_{K} \sigma$ on the vertex set $[m]$.

A simplex $\tau$ on the vertex set $[m]$ is not a simplex of $\partial \operatorname{star}_{K} \sigma=\operatorname{star}_{K} \sigma \backslash$ Intstar $_{K} \sigma$ if and only if $\tau \in$ Intstar $_{K} \sigma$ or $\tau \notin \operatorname{star}_{K} \sigma$, i.e. $\sigma \subset \tau$ or there exists a $\sigma_{i} \backslash \sigma$ such that $\sigma_{i} \backslash \sigma \subset \tau$, so

$$
2^{[m]} \backslash \partial \operatorname{star}_{K} \sigma=2^{[m]} \backslash K_{\{\sigma, \mathbb{P}-\sigma\}}([m]),
$$

$\{\sigma, \mathbb{P}-\sigma\}$ is a simplicial complement of $\partial \operatorname{star}_{K} \sigma$ on the vertex set $[m]$.

Take the cone of $\partial \operatorname{star}_{K} \sigma$ on the vertex set $[m+1]$, a simplex $\tau \subset[m]$ or $(\tau, m+$ 1) $\subset[m+1]$ is not a simplex of conedstar $_{K} \sigma=(m+1) * \partial \operatorname{star}_{K} \sigma$ if and only if $\tau$ is not a simplex of $\partial \operatorname{star}_{K} \sigma$, i.e.

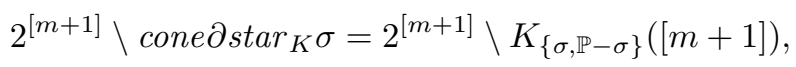

$\{\sigma, \mathbb{P}-\sigma\}$ is a simplicial complement of conedstar $_{K} \sigma$ on the vertex set $[m+1]$. 
Second, we prove that $\{\mathbb{P}, \sigma,(m+1)\}$ is a simplicial complement of $K \backslash$ Intstar $_{K} \sigma$ on the vertex set $[m+1]$.

A simplex $\tau$ on the vertex set $[m]$ is not a simplex of $K \backslash$ Intstar $_{K} \sigma$ if and only if $\tau \notin K$ or $\tau \in$ Intstar $_{K} \sigma$, i.e. there exists a $\sigma_{i} \in \mathbb{P}$ such that $\sigma_{i} \subset \tau$ or $\sigma \subset \tau$. $\{\mathbb{P}, \sigma\}$ is a simplicial complement of $K \backslash$ Intstar $_{K} \sigma$ on the vertex set $[m]$.

Consider $K \backslash$ Intstar $_{K} \sigma$ as a simplicial complex on the vertex set $[m+1],(m+1)$ does not appear in $K \backslash \operatorname{Intstar}_{K} \sigma$. It is a ghost vertex and $(m+1)$ is a missing face. So

$$
\{\mathbb{P}, \sigma,(m+1)\}
$$

is a simplicial complement of $K \backslash$ Intstar $_{K} \sigma$ on the vertex set $[m+1]$.

From Lemma 2.7, we know that $\{\mathbb{P}, \sigma,(m+1)\} *\{\sigma, \mathbb{P}-\sigma\}$ is a simplicial complement of $s s_{\sigma} K=\left(K \backslash\right.$ Intstar $\left._{K} \sigma\right) \cup\left(\right.$ conedstar $\left._{K} \sigma\right)$, where

$$
\{\mathbb{P}, \sigma,(m+1)\} *\{\sigma, \mathbb{P}-\sigma\}=\left\{\begin{array}{ll}
\mathbb{P} * \sigma, & \mathbb{P} *\{\mathbb{P}-\sigma\}, \\
\sigma * \sigma, & \sigma *\{\mathbb{P}-\sigma\}, \\
(m+1) * \sigma, & (m+1) *\{\mathbb{P}-\sigma\}
\end{array}\right\} .
$$

At last, we complete the proof by showing that the simplicial complement $\{\mathbb{P}, \sigma$, $(m+1)\} *\{\sigma, \mathbb{P}-\sigma\}$ is equivalent to $\{\mathbb{P}, \sigma,\{\mathbb{P}-\sigma\} *(m+1)\}$, i.e.

$$
s s_{\sigma} K=K_{\{\mathbb{P}, \sigma,(m+1)\} *\{\sigma, \mathbb{P}-\sigma\}}([m+1])=K_{\{\mathbb{P}, \sigma,\{\mathbb{P}-\sigma\} *(m+1)\}}([m+1]),
$$

First,

$$
\sigma * \sigma=\sigma \in\{\mathbb{P}, \sigma,(m+1)\} *\{\sigma, \mathbb{P}-\sigma\} .
$$

Every subset $\sigma_{i} \cup \sigma \in \mathbb{P} * \sigma,(\sigma, m+1) \in(m+1) * \sigma$ and $\sigma \cup\left(\sigma_{i} \backslash \sigma\right) \in \sigma *\{\mathbb{P}-\sigma\}$ contain $\sigma$. They could be removed from $\{\mathbb{P}, \sigma,(m+1)\} *\{\sigma, \mathbb{P}-\sigma\}$, so

$$
\{\mathbb{P}, \sigma,(m+1)\} *\{\sigma, \mathbb{P}-\sigma\} \simeq\left\{\begin{array}{l}
\mathbb{P} *\{\mathbb{P}-\sigma\} \\
\sigma, \\
(m+1) *\{\mathbb{P}-\sigma\}
\end{array}\right\} .
$$

Then for any $\sigma_{i} \in \mathbb{P}$, one has $\sigma_{i} \backslash \sigma \in \mathbb{P}-\sigma$. So

$$
\sigma_{i}=\sigma_{i} \cup\left(\sigma_{i} \backslash \sigma\right) \in \mathbb{P} *\{\mathbb{P}-\sigma\} .
$$

Any other $\sigma_{i} \cup\left(\sigma_{j} \backslash \sigma\right) \in \mathbb{P} *\{\mathbb{P}-\sigma\}$ contains $\sigma_{i}$, they could be removed from $\mathbb{P} *$ $\{\mathbb{P}-\sigma\}$. Thus $\mathbb{P} *\{\mathbb{P}-\sigma\}$ is equivalent to $\mathbb{P}$ and $\{\mathbb{P}, \sigma,(m+1)\} *\{\sigma, \mathbb{P}-\sigma\}$ could be reduced to

$$
\{\mathbb{P}, \sigma,\{\mathbb{P}-\sigma\} *(m+1)\}=\left\{\mathbb{P}, \sigma,\left(\sigma_{1} \backslash \sigma, m+1\right),\left(\sigma_{2} \backslash \sigma, m+1\right), \ldots,\left(\sigma_{s} \backslash \sigma, m+1\right)\right\} .
$$

The theorem follows.

Remark 2.10. If $\sigma$ is not a simplex of $K$, we still have $\{\mathbb{P}, \sigma,\{\mathbb{P}-\sigma\} *(m+1)\}$ as a simplicial complement of a simplicial complex $s s_{\sigma} K$. In that case, there exists a $\sigma_{i} \in \mathbb{P}$ such that $\sigma_{i} \subseteq \sigma$. So $\sigma$ could be removed from $\{\mathbb{P}, \sigma,\{\mathbb{P}-\sigma\} *(m+1)\}$ and $\sigma_{i} \backslash \sigma=\emptyset \in \mathbb{P}-\sigma$. Thus $\left(\sigma_{i} \backslash \sigma, m+1\right)=(m+1) \in\{\mathbb{P}-\sigma\} *(m+1)$ and all the other $\left(\sigma_{j} \backslash \sigma, m+1\right)$ could be removed from $\{\mathbb{P}, \sigma,\{\mathbb{P}-\sigma\} *(m+1)\}$. That is to say 
that $(m+1)$ is a missing face and

$$
\{\mathbb{P}, \sigma,\{\mathbb{P}-\sigma\} *(m+1)\} \simeq\{\mathbb{P},(m+1)\}
$$

is still a simplicial complement of $s s_{\sigma} K=K$ but on the vertex set $[m+1]$ and a ghost vertex $(m+1)$ is added.

We still call it the stellar subdivision at $\sigma$ on $K$.

Example 2.11. In Example 2.4, we make stellar subdivision at $\sigma=(1,2)$ on $K$ (see Figure 2).
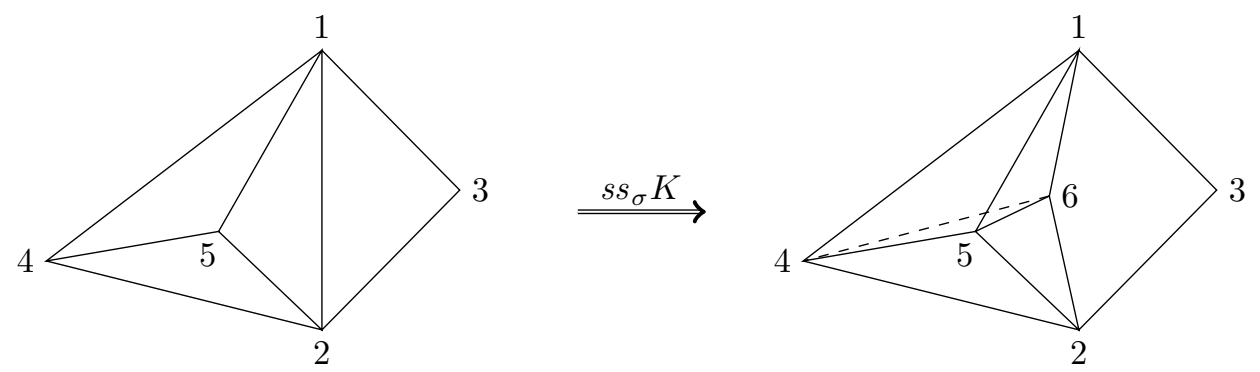

Figure 2: The stellar subdivision at $\sigma$ on $K$

$\mathbb{P}=\{(1,2,4,5),(1,2,3),(3,4),(3,5)\}$ is a simplicial complement of $K, \sigma=(1,2)$, so

$$
\begin{gathered}
\{\mathbb{P}-\sigma\} *(6)=\{(4,5),(3),(3,4),(3,5)\} *(6)=\{(4,5,6),(3,6),(3,4,6),(3,5,6)\} . \\
\{\mathbb{P}, \sigma,\{\mathbb{P}-\sigma\} *(6)\}=\left\{\begin{array}{l}
(1,2,4,5),(1,2,3),(3,4),(3,5), \\
(1,2)=\sigma, \\
(4,5,6),(3,6),(3,4,6),(3,5,6)
\end{array}\right\}
\end{gathered}
$$

is a simplicial complement of $s s_{\sigma} K$. The maximal simplices of $s s_{\sigma} K$ are

$$
\left\{\begin{array}{l}
(1,3),(2,3),(1,4,5),(2,4,5) \\
(1,4,6),(1,5,6),(2,4,6),(2,5,6)
\end{array}\right\}
$$

\section{Construction}

After given the simplicial complement of stellar subdivision, we construct our moment-angle manifolds whose cohomology has torsion.

Lemma 3.1. Let $K$ be a simplicial complex on the vertex set $[m]$ and

$$
\mathbb{P}=\left\{\sigma_{1}, \sigma_{2}, \ldots, \sigma_{s}\right\}
$$

be a simplicial complement of it. Let $I$ be a subset of the vertex set $[m]$. Then

$$
\left.\mathbb{P}\right|_{I}=\left\{\sigma_{i} \in \mathbb{P} \mid \sigma_{i} \subset I\right\}
$$

is a simplicial complement of the full subcomplex $\left.K\right|_{I}$ on the vertex set $I$. 
Proof. From its definition, we know that the full subcomplex

$$
\left.K\right|_{I}=\{\sigma \in K \mid \sigma \subset I\}
$$

is a simplicial complex on the vertex set $I$. A subset $\tau$ on the vertex set $I$ is not a simplex of $\left.K\right|_{I}$ if and only if $\tau$ is not a simplex of $K$, i.e. there exists a non-face $\sigma_{i} \in \mathbb{P}$ such that $\tau_{i} \subset \tau$. Note that $\tau \subset I, \tau_{i} \subset \tau \subset I$. The lemma follows.

Theorem 3.2 (Construction). Let $K$ be a subcomplex (not a full subcomplex) of a simplicial sphere $L_{0}$ on the vertex set $[m], \mathbb{M}=\left\{\sigma_{1}, \sigma_{2}, \ldots, \sigma_{s}\right\}$ be the set of missing faces of $K$, which are also simplices of $L_{0}$. On $L_{0}$, make stellar subdivisions at $\sigma_{1}, \sigma_{2}, \ldots, \sigma_{s}$ one by one as follows

$$
L_{1}=s s_{\sigma_{1}} L_{0}, \quad L_{2}=s s_{\sigma_{2}} L_{1}, \quad \ldots, \quad L_{s}=s s_{\sigma_{s}} L_{s-1} .
$$

Then $K$ becomes a full subcomplex of $L_{s}, K=\left.L_{s}\right|_{[m]}$.

Proof. Let $\mathbb{P}_{0}=\left\{\tau_{1}, \tau_{2}, \ldots, \tau_{r}\right\}$ be a simplicial complement of $L_{0}$ on $[m]$. From Theorem 2.9 we know that

$$
\mathbb{P}_{1}=\left\{\mathbb{P}_{0}, \sigma_{1}, \mathbb{P}_{1}^{\prime}\right\}
$$

is a simplicial complement of $L_{1}=s s_{\sigma_{1}} L_{0}$ on $[m+1]$, where

$$
\mathbb{P}_{1}^{\prime}=\left\{\mathbb{P}_{0}-\sigma_{1}\right\} *(m+1) .
$$

By induction, we get a simplicial complement of $L_{s}=s s_{\sigma_{s}} L_{s-1}$ on $[m+s]$ as

$$
\begin{aligned}
\mathbb{P}_{s} & =\left\{\mathbb{P}_{s-1}, \sigma_{s}, \mathbb{P}_{s}^{\prime}\right\} \\
& =\left\{\mathbb{P}_{0}, \sigma_{1}, \sigma_{2}, \ldots, \sigma_{s}, \mathbb{P}_{1}^{\prime}, \mathbb{P}_{2}^{\prime}, \ldots, \mathbb{P}_{s}^{\prime}\right\},
\end{aligned}
$$

where

$$
\mathbb{P}_{i}^{\prime}=\left\{\mathbb{P}_{i-1}-\sigma_{i}\right\} *(m+i)
$$

Note that every non-face in $\mathbb{P}_{i}^{\prime}$ contains $(m+i)$ as a vertex. From Lemma 3.1 we know that

$$
\left.\mathbb{P}_{s}\right|_{[m]}=\left\{\mathbb{P}_{0}, \sigma_{1}, \sigma_{2}, \ldots, \sigma_{s}\right\}
$$

is a simplicial complement of the full subcomplex $\left.L_{s}\right|_{[m]}$.

Finally, we consider the simplicial complement $\left.\mathbb{P}_{s}\right|_{[m]}$. Note that $K$ is a subcomplex of $L_{0}$, every non-face $\tau_{i} \in \mathbb{P}_{0}$ is not a simplex of $K$, so there exists a $\sigma_{j} \in \mathbb{M}$ such that $\sigma_{j} \subseteq \tau_{i}$. Then $\tau_{i}$ could be removed from $\left\{\mathbb{P}_{0}, \sigma_{1}, \sigma_{2}, \ldots, \sigma_{s}\right\}$.

Thus

$$
\left.\mathbb{P}_{s}\right|_{[m]}=\left\{\mathbb{P}_{0}, \sigma_{1}, \sigma_{2}, \ldots, \sigma_{s}\right\} \simeq\left\{\sigma_{1}, \sigma_{2}, \ldots, \sigma_{s}\right\}=\mathbb{M}
$$

which is the set of missing faces of $K$. The theorem follows.

Remark 3.3. If $L_{0}$ is also a polytopal sphere, the stellar subdivision of $L_{0}$ is also polytopal. It has been proved in a geometric sense by Ewald and Shephard in [10].

Let $\widetilde{L}_{0}$ be the simplicial polytope and its boundary $\partial \widetilde{L}_{0}=L_{0}$ be the polytopal sphere. If $\sigma$ is a simplex of $L_{0}$ and $\sigma$ is the intersection of the facets (maximal simplices of $\left.L_{0}\right) F_{i_{1}}, F_{i_{2}}, \ldots, F_{i_{r}}$, one can take any point $p$ beyond the facets $F_{i_{1}}, F_{i_{2}}, \ldots, F_{i_{r}}$ and beneath the other facets (See [11, p. 78] for the definitions of "beyond" and 
"beneath"). The stellar subdivision $s s_{\sigma} \partial \widetilde{L}_{0}$ is the boundary of the convex hall of $\widetilde{L}_{0}^{\prime}=\operatorname{conv}\left(\widetilde{L}_{0} \cup p\right)$.

It could also be proved from the duality of polytopes.

Let $\widetilde{L}_{0}$ be the simplicial polytope corresponding to $L_{0}$, and $P_{0}$ be the dual simple polytope, (the vertex of $L_{0}$ corresponding to the facet while the facet of $L_{0}$ corresponding to the vertex of $\left.P_{0}\right)$. Let $\sigma=\left(i_{1}, i_{2}, \ldots, i_{k}\right)$ be a simplex of $L_{0}$, make a stellar subdivision at $\sigma$ on $L_{0}$ is equivalent, though the duality of polytopes, to cutting off the face $\sigma^{*}=F_{i_{1}} \cap F_{i_{2}} \cap \cdots \cap F_{i_{k}}$ in $P_{0}$ by a generic hyperplane. The cutting off operation on a simple polytope is still simple, so $s s_{\sigma} \partial \widetilde{L}_{0}$ is polytopal.

\section{Application}

Proposition 4.1. The cohomology of differentiable moment-angle manifolds may have torsion of any order.

Proof. Let $L_{0}$ be a polytopal sphere and $K$ be a subcomplex of $L_{0}$, whose cohomology has torsion. Construct a new polytopal sphere $L_{s}$ by Theorem 3.2, then $K$ becomes a full subcomplex of $L_{s}$, while both $\mathbb{R} \mathcal{Z}_{L_{s}}$ and $\mathcal{Z}_{L_{s}}$ are framed differentiable manifolds. From Hochster's Theorem, the cohomology of $\mathbb{R} \mathcal{Z}_{L_{s}}$ and $\mathcal{Z}_{L_{s}}$ has $\widetilde{H}^{*}(K)$ as a summand and then have torsion.

At least, every simplicial complex $K$ with $m$ vertexes is a subcomplex of the polytopal sphere $\partial \Delta^{m-1}$. So the cohomology of differentiable moment-angle manifolds could have any torsion.

Here is an example.

Example 4.2. Let $K$ be the triangulated mod 3 Moore space (see Figure 3) which can be embedded in 6-dimensional polytopal sphere

$$
L_{0}=\partial \Delta^{7}=\partial(1,2,3,4,5,6,7,8) .
$$

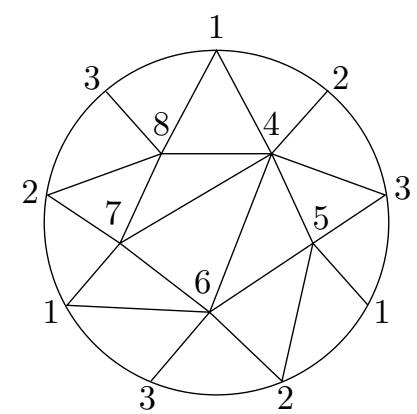

Figure 3: The triangulated mod 3 Moore space

The set of missing faces of $L_{0}$ is

$$
\mathbb{P}_{0}=\{(1,2,3,4,5,6,7,8)\} .
$$


The set of missing faces of $K$ is

$$
\mathbb{M}=\left\{\begin{array}{l}
(1,2,3),(1,2,6),(1,2,8),(1,3,4), \\
(1,4,5),(1,4,6),(1,4,7),(1,5,6),(1,7,8), \\
(2,3,5),(2,4,5),(2,4,6),(2,4,7),(2,4,8),(2,6,7), \\
(3,4,6),(3,4,8),(3,5,6), \\
(3,7),(5,8),(5,7),(6,8)
\end{array}\right\}
$$

and the set of maximal simplices of $\mathrm{K}$ is

$$
\left\{\begin{array}{l}
(1,2,4),(1,2,5),(1,2,7),(1,3,5), \\
(1,3,6),(1,3,8),(1,4,8),(1,6,7), \\
(2,3,4),(2,3,6),(2,3,8),(2,5,6),(2,7,8), \\
(3,4,5),(4,5,6),(4,6,7),(4,7,8)
\end{array}\right\} .
$$

Making 22 stellar subdivisions at missing faces of $K$ on $\partial \Delta^{7}$, we thus obtain a 6dimensional polytopal sphere $L_{22}$ with 30 vertices which has $K$ as a full subcomplex. The real moment-angle manifold corresponding to $L_{22}$ is of 6-dimensional while the complex one is of 37-dimensional where $H^{3}\left(\mathbb{R}_{\mathcal{Z}_{22}}\right)$ and $H^{11}\left(\mathcal{Z}_{L_{22}}\right)$ has $\widetilde{H}^{2}(K)=\mathbb{Z} / 3$ as a summand.

Passing to the dual, $\Delta^{7}$ is the dual simple polytope of $\partial \Delta^{7}$ with facets numbered as vertexes of $\partial \Delta^{7}$. Making stellar subdivision on $\partial \Delta^{7}$ at $\sigma=\left(i_{1}, i_{2}, \ldots, i_{r}\right)$ is dual to cutting off face $F_{i_{1}} \cap F_{i_{2}} \cap \cdots \cap F_{i_{r}}$ in $\Delta^{7}$,

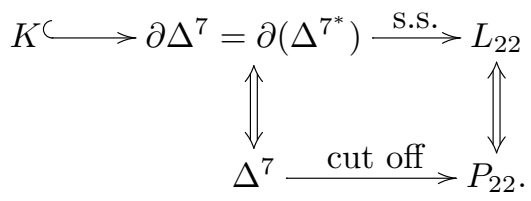

After cutting off the faces $F_{i_{1}} \cap F_{i_{2}} \cap \cdots \cap F_{i_{r}}$ numbered at $\mathbb{M}$ in (2), one gets a simple polytope $P_{22}$. The cohomology of the moment-angle manifold corresponding to $P_{22}$ has $H^{2}(K)=\mathbb{Z} / 3$ as a summand and then has torsion. If we only cut off $\{1,2, \ldots, 8\} \backslash \sigma$ for every maximal simplex $\sigma$ of $K$ in $\Delta^{7}$ as Bosio and Meersseman did in [2, Theorem 11.12], we do not get torsion.

Compute the missing faces after making stellar subdivision at $(1,2,3)$ and $(3,7)$ on $\partial \Delta^{7}$ in different sequence, one has

1. We make stellar subdivision at $(1,2,3)$ on $L_{0}=\partial \Delta^{7}$ at first, then make stellar subdivision at $(3,7)$.

From Theorem 2.9 we know that,

$$
\begin{aligned}
\mathbb{P}_{0} & =\{(\underline{(1,2,3,4,5,6,7,8)}\}, \\
\sigma_{1} & =(1,2,3), \\
\left(\mathbb{P}_{0}-\sigma_{1}\right) *(9) & =\{(4,5,6,7,8,9)\}
\end{aligned}
$$

is a simplicial complement of $L_{1}=s s_{(1,2,3)} L_{0}$. After removing the larger nonfaces $(1,2,3,4,5,6,7,8)$, we get the set of missing faces of $L_{1}$

$$
\mathbb{M}_{1}=\{(1,2,3),(4,5,6,7,8,9)\} .
$$

Then we make stellar subdivision at $(3,7)$ on $L_{1}$ and get the set of missing faces 


$$
\text { of } \begin{aligned}
L_{2}= & s s_{(3,7)} L_{1} \\
& \mathbb{M}_{2}=\{(1,2,3),(4,5,6,7,8,9),(3,7),(1,2,10),(4,5,6,8,9,10)\} .
\end{aligned}
$$

2. Similarly, we make stellar subdivision at $(3,7)$ on $L_{0}$ at first, then make stellar subdivision at $(1,2,3)$, the resulting set of missing faces of $L_{2}^{\prime}$ is

$$
\mathbb{M}_{2}^{\prime}=\{(3,7),(1,2,4,5,6,8,9),(1,2,3),(7,10),(4,5,6,8,9,10)\} .
$$

It is easy to see that two simplicial complexes $K$ and $K^{\prime}$ on the vertex set $I$ are combinatorially equivalent if and only if their sets of missing faces $\mathbb{M}$ and $\mathbb{M}^{\prime}$ are equivalent, i.e. there exists a one to one correspondence $\phi: I \rightarrow I$ that gives a one to one correspondence between $\mathbb{M}$ and $\mathbb{M}^{\prime}$.

Comparing with these two sequences, we can find that $L_{2}$ has one 2 -vertex missing faces $(3,7)$ while $L_{2}^{\prime}$ has two $(3,7),(7,10)$. This implies that $L_{2}$ is not combinatorially isomorphic to $L_{2}^{\prime}$ and this difference might persist during the later stellar subdivisions.

Remark 4.3. Though $K$ will be a full subcomplex of $L_{s}$ in every sequence of making stellar subdivisions at $K$ 's missing faces, the combinatorial structure of $L_{s}$ may not be combinatorially isomorphic in different sequences.

\section{References}

[1] I.V. Baskakov, Cohomology of $K$-powers of spaces and the combinatorics of simplicial divisions, Uspekhi Mat. Nauk 57 (2002), no. 5, 147-148 (Russian), Russian Math. Surveys, 57 (2002), no. 5, 898-990 (English translation).

[2] F. Bosio and L. Meersseman, Real quadrics in $\mathbb{C}^{n}$, complex manifolds and convex polytopes, Acta Math. 197 (2006), no. 1, 53-127.

[3] V.M. Buchstaber and T.E. Panov, Torus actions and their applications in topology and combinatorics, Univ. Lecture Ser., vol. 24, Amer. Math. Soc., Providence, RI, 2002.

[4] V.M. Buchstaber and T.E. Panov, Toric topology, Math. Surveys Monogr., vol. 204, Amer. Math. Soc., Providence, RI, 2015.

[5] V.M. Buchstaber, T.E. Panov and N. Ray, Spaces of polytopes and cobordism of quasitoric manifolds, Mosc. Math. J. 7 (2007), no. 2, 219-242.

[6] V.M. Buchstaber and N. Ray, Tangential structures on toric manifolds, and connected sums of polytope, Int. Math. Res. Not. IMRN (2001), no. 4, 193219.

[7] L. Cai, Norm minima in certain Siegel leaves, Algebr. Geom. Topol. 15 (2015), no. $1,445-466$.

[8] L. Cai and S. Choi, On the topology of a small cover associated to a shellable complex, preprint (2016) arXiv:1604.06988

[9] S. Choi and H. Park, On the cohomology and their torsion of real toric objects, Forum Math. 29 (2017), no. 3, 543-553

[10] G. Ewald and G.C. Shephard, Stellar Subdivisions of Boundary Complexes of Convex Polytopes, Math. Ann. 210 (1974), 7-16 
[11] B. Grünbaum, Convex Polytopes, Grad. Texts in Math., vol. 221 SpringerVerlag 2003.

[12] J.R. Munkres, Elements of Algebraic Topology, Addision-Wesley Publishing Company 1984.

[13] X. Wang and Q. Zheng, The homology of simplicial complements and the cohomology of polyhedral products, Forum Math. 27 (2015), no. 4, 2267-2299.

Xiaomeng Li lxmnku1992@163.com

Henan Experimental High School, Zhengzhou, Henan 450000, P. R. China

Gefei Wang 1412640@mail.nankai.edu.cn

School of Mathematical Science, Nankai University, Tianjin 300071, P. R. China 